\title{
Interference Allowance in Clustered Joint Processing and Power Allocation
}

\author{
E. Katranaras, D. Kaltakis, M. A. Imran and R. Hoshyar \\ Centre for Communication Systems Research CCSR \\ University of Surrey, UK \\ Email: e.katranaras@surrey.ac.uk
}

\begin{abstract}
We derive an analytical formula for the sum rate of the uplink of a linear network of cells when clustered joint processing is adopted among the base stations in a generalised fading environment. An inter-cluster interference allowance scheme is considered and various user power allocation profiles are investigated in terms of optimal achievable sum rate to highlight that cell-based power allocation is preferable to cluster-based. The contribution of each base station on the cluster sum rate is investigated and its importance is discussed. Numerical results are produced for a real-world scenario showing how medium density systems are the most viable case for clustered system design by achieving $>80 \%$ of the global cooperation capacity.
\end{abstract}

\section{INTRODUCTION}

Communication systems are evolving day by day and the increasing demand for high data rate services has rendered the investigation for rate limits extremely important. The first concrete findings in this regard ([1],[2],[3]) raised the significance of cooperation between all Base Station (BS) receivers in the uplink channel to jointly process the signals at a centralised receiver - termed as a Hyper Receiver (HR) via unlimited rate links. Although optimal, from the information theoretic system rate point of view, the joint processing of all the BSs in the system is very hard to be implemented in real world, mainly because of the large distances between them. The concept of clustered multi-cell cooperative processing, to decentralize the joint decoding of all the users in the system, has attracted a lot of attention recently and is adopted in numerous investigations with some of them attempting to determine achievable rates or upper bounds ([4], [5]).

The principal focus of this research will be on the effect of user power allocation on the achievable rate of a clustering scheme and to determine how close these rates are to the capacity of the HR scheme. A first attempt to elaborate on the effect of user power control in the uplink of a clustered systems was made in [6]. Here, we overcome the assumption of adjacent cell interference and we investigate the user power allocation along with the sum rate optimisation problem in greater depth. These findings will give important insights on the achievable bounds of cooperative multi-cell networks and determine if clustering with user power control is useful alternative to the hard to implement HR scheme.

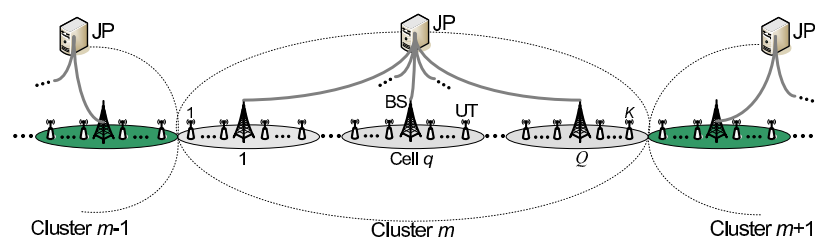

Fig. 1. Linear clustered cellular system model.

\section{SySTEM MODEL}

We investigate a similar system model as the one presented in [4], [6] focusing on the uplink of a cellular system. Consider a linear system of $N$ cells, divided into $N_{Q}$ smaller networks (clusters of cells) each with $Q \ll N$ cells. The BSs are uniformly distributed across a linear grid, each one at the centre of each cell. $K$ UTs are distributed across each cell. The cooperation among the BSs is limited only to those in cells that belong to the same cluster and hence a Joint Processor (JP) in each cluster jointly decodes all the UTs received signals of that cluster (Figure 1). An inter-cluster interference allowance scheme with no isolation between clusters is considered and UTs in all clusters are allowed to exploit the full resources allocated to the system. The cells of every cluster experience inter-cluster interference as their BSs will be interfered by transmitted signals from the users in the other clusters.

The path loss and fading models that are presented in [7], [8] are also employed here. The path gain (defined as the ratio of the received over the transmitted power) is mapped with the distance in a power-law path loss environment as:

$$
\varsigma_{k \dot{q} \dot{m}}^{m, q}=\sqrt{L_{0}}\left(1+D_{\dot{m}, \dot{q}, k}^{m, q}\right)^{-\eta / 2}
$$

where $L_{0}$ is defined as the power received at a reference distance when transmitted power is unity and $\eta$ is the powerlaw path loss exponent. $D_{\dot{m}, \dot{q}, k}^{m, q}$ is defined as the distance between user $k$ in cell $\dot{q}$ of cluster $\dot{m}$ from the reference point in cell $q$ of cluster $m$. Moreover, considering the uniformly distributed random received phase $\Phi_{\dot{m}, \dot{q}, k}^{m, q}$ on the specular path between transmitter $k$ in cell $\dot{q}$ of cluster $\dot{m}$ and the BS of cell $q$ of cluster $m$, a generalised model for the fading coefficients can be given by [9], [10], [11]:

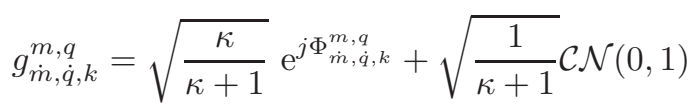


where $\mathbb{E}[g g *]=1, \kappa$ is the ratio of the power in the specular path and the non-specular multipaths and $\mathcal{C N}(0,1)$ represents a complex Gaussian random variable with independent real and imaginary components each normally distributed with zero mean and variance of $1 / 2$.

\section{Sum RATE ANALysis}

To facilitate sum rate analysis, we assume symmetry among all clusters of cells and we omit the system edge effects. In that case, analysis can be performed only for one cluster and the results will be valid for all the clusters of the cellular system. The received signal $y^{m, q}$ at the BS in cell $q$ of cluster $m$ is the sum of the transmitted signals $x_{m, \dot{q}, k}$ of all the UTs in the same cluster of cells appropriately scaled by each channel gain coefficient, plus the sum $z^{m, q}$ of the thermal AWGN $\left(n^{m, q}\right.$ with $\left.n \sim \mathcal{C N}\left(0, \sigma_{0}^{2}\right)\right)$ and the interfering complex Gaussian inputs from UTs in cells outside the cluster of interest. Thus:

$$
y^{m, q}=\sum_{\dot{q}=1}^{Q} \sum_{k=1}^{K} \varsigma_{m, \dot{q}, k}^{m, q} g_{m, \dot{q}, k}^{m, q} x_{m, \dot{q}, k}+z^{m, q}
$$

The undesired signal $z^{m, q}$ can be given as

$$
z^{m, q}=n^{m, q}+\sum_{\dot{m}} \sum_{\dot{q}=1}^{Q} \sum_{k=1}^{K}\left[\varsigma_{\dot{m}, \dot{q}, k}^{m, q} g_{\dot{m}, \dot{q}, k}^{m, q} x_{\dot{m}, \dot{q}, k}\right]
$$

where $\dot{m} \neq m$. Inter-cluster interference, since it is a sum of complex Gaussian inputs, can be viewed as additional AWGN component at the BSs and hence, $z^{m, q}$ can still be considered AWGN with power given by

$$
\begin{aligned}
Z^{m, q} & =\mathbb{E}\left[z^{m, q} \cdot\left(z^{m, q}\right)^{*}\right]=\sigma_{0}^{2}+\sum_{\dot{m}} \sum_{\dot{q}=1}^{Q} \sum_{k=1}^{K} \\
\mathbb{E} & {\left[\left(\varsigma_{\dot{m}, \dot{q}, k}^{m, q} g_{\dot{m}, \dot{q}, k}^{m, q} x_{\dot{m}, \dot{q}, k}\right)\left(\varsigma_{\dot{m}, \dot{q}, k}^{m, q} g_{\dot{m}, \dot{q}, k}^{m, q} x_{\dot{m}, \dot{q}, k}\right)^{*}\right] }
\end{aligned}
$$

where $\sigma_{0}^{2}$ is thermal AWGN power at the receiver end.

Consider $\mathbf{x}=\left[\mathbf{x}_{m, 1^{T}}, \mathbf{x}_{m, 2}{ }^{T}, \ldots \mathbf{x}_{m, Q}\right]^{T}$ to be the $Q K \times 1$ vector of the transmitted signals of all the UTs in cluster $m$, with $\mathbf{x}_{m, q}=\left[x_{m, q, 1}, \ldots, x_{m, q, K}\right]^{T}$ denoting the concatenation of the transmitted signals from the $K$ UTs in cell $q, \mathbf{z}$ to be the $Q \times 1$ column noise vector and $\mathbf{H}$ to be the overall $Q \times$ $Q K$ cluster channel gain matrix. Following the information theoretic analysis described in [6] a tight upper bound for the maximum achievable cluster sum rate can be given by:

$$
\begin{aligned}
R= & \mathbb{E}_{\mathbf{H}}\left[\log \left(\frac{\operatorname{det}\left(\mathbf{H} \boldsymbol{\Lambda}_{\mathbf{x}} \mathbf{H}^{\dagger}+\boldsymbol{\Lambda}_{\mathbf{z}}\right)}{\operatorname{det}\left(\boldsymbol{\Lambda}_{\mathbf{z}}\right)}\right)\right] \\
& \cong \log \left(\frac{\operatorname{det} \mathbb{E}_{\mathbf{H}}\left[\mathbf{H} \boldsymbol{\Lambda}_{\mathbf{x}} \mathbf{H}^{\dagger}+\boldsymbol{\Lambda}_{\mathbf{z}}\right]}{\operatorname{det}\left(\boldsymbol{\Lambda}_{\mathbf{z}}\right)}\right) \text { for } K \rightarrow \infty
\end{aligned}
$$

where the expectation (indicated by subscript $\mathbf{H}$ ) is taken over all the system fading realizations, $\boldsymbol{\Lambda}_{(\cdot)}$ stands for the covariance matrix of the respective vector and the convergence is due to the law of large numbers [2], [8].

\section{A. UT Power Allocation}

One way to reduce inter-cluster interference is to perform power control on the UTs of the system. We assume that all UT signals during a long enough time period experience all possible fading states. Hence, the parameter that defines the strength of a signal over that period is the UT location. For that reason, we consider a variable UT power allocation according to the instantaneous position of each UT to its respective BS and cluster. Due to the cluster symmetry and the same UT distribution at each cell, the power allocation will be the same at any cluster. Hence, we omit the cluster index at the power symbolisation. The input covariance matrix, since we assume independent inputs, will be a diagonal matrix of $Q K$ elements:

$$
\boldsymbol{\Lambda}_{\mathbf{x}}=\operatorname{diag}(\overbrace{P_{1,1} \ldots P_{q, k} \ldots P_{Q, K}}^{Q K})
$$

where $P_{q, k}$ denotes the power of UT $k$ in cell $q$ of any cluster. Furthermore, from (5) the noise power matrix will be:

$$
\boldsymbol{\Lambda}_{\mathbf{z}}=\operatorname{diag}(\overbrace{\ldots \sigma_{0}^{2}+\sum_{\dot{m}} \sum_{\dot{q}=1}^{Q} \sum_{k=1}^{K} P_{\dot{q}, k}\left(\varsigma_{\dot{m}, \dot{q}, k}^{m, q}\right)^{2} \ldots}^{Q})
$$

By substituting (7) and (8) in (6) and recalling that the determinant of a matrix is the product of its eigenvalues we have (for $K \rightarrow \infty$ ):

$$
R \cong \log \prod_{q=1}^{Q}\left[1+\frac{\sum_{\dot{q}=1}^{Q} \sum_{k=1}^{K} P_{\dot{q}, k}\left(\varsigma_{m, \dot{q}, k}^{m, q}\right)^{2}}{\sigma_{0}^{2}+\sum_{\dot{m}} \sum_{\dot{q}=1}^{Q} \sum_{k=1}^{K} P_{\dot{q}, k}\left(\varsigma_{\dot{m}, \dot{q}, k}^{m, q}\right)^{2}}\right]
$$

To comprehend the derivation of (9) we remark that at the diagonal entries of $\mathbb{E}_{\mathbf{H}}\left[\mathbf{H} \boldsymbol{\Lambda}_{\mathbf{x}} \mathbf{H}^{\dagger}\right]$ the product that takes place is $\mathbb{E}_{g}[g g *]=1$ while for the off-diagonal entries we have the expectation of the product of two different realisations of the fading coefficients $\mathbb{E}_{g}\left[g(\dot{g})^{*}\right]=0$, indicating that $\mathbb{E}_{\mathbf{H}}\left[\mathbf{H} \boldsymbol{\Lambda}_{\mathbf{x}} \mathbf{H}^{\dagger}\right]$ converges to a $Q \times Q$ diagonal matrix [7].

\section{BS CONTRIBUtion ON SUm RATE AND RoT DISTRIBUTION}

The cluster sum rate given in (9) can be written as:

$$
R=\sum_{q=1}^{Q} \log \left[1+\operatorname{RoT}_{q}\right]
$$

with The Rise over Thermal referring to a BS of cell $q\left(\operatorname{RoT}_{q}\right)$ is defined as:

$$
\operatorname{RoT}_{q} \triangleq \frac{\text { Total Desired Received Power }}{\text { Total Undesired Received Power }}=\frac{P^{m, q}}{Z^{m, q}}
$$

where $P^{m, q}$ is the intra-cluster received power from UTs within the region of the respective JP and $Z^{m, q}$ is the total undesired power at cell $q$ of cluster $m$ given in (5). Note that $\operatorname{RoT}_{q}$ actually refers to the $q^{\text {th }}$ individual eigenvalue of (9). 
Based on the cell RoT definition in (11), we define the contribution of receiver $q$ on the cluster sum rate as $R_{q} \triangleq$ $\log \left(1+\operatorname{RoT}_{q}\right)$. Considering that, we can obtain a distribution on the contribution rates of each receiver according to the individual cell RoT distribution. In the following along with the cluster sum rate we investigate the contributing rates of the BSs in the cluster. The distribution of the BS contribution rates does not necessarily provide information about the percell sum rate (sum of all user rates in cell) distribution or cell rate fairness of the system but it will help us on tackling the sum rate optimization problem and in any case it may stand for a useful rate metric for the clustered systems as it shows which BS contributes less or more on the cluster sum rate.

\section{Sum Rate Optimization}

Our aim is to investigate on the optimal power allocation $\mathbf{p}^{\star}=\left(P_{1,1}^{\star}, P_{1,2}^{\star}, \ldots, P_{Q, K}^{\star}\right)$ so as the cluster sum rate $R$ is maximized under given system power constraints. A maximum $\left(P_{\max }\right)$ as well as a minimum UT power constraint $\left(P_{\min }\right)$ is considered. We determine

$$
\mathbf{p}^{\star}=\arg \max _{\mathbf{p} \in\left\{\mathbf{p} \mid P_{\min } \leq P_{q, k} \leq P_{\max }, \forall(q, k)\right\}} R(\mathbf{p})
$$

where the set $\left\{\mathbf{p} \mid P_{\min } \leq P_{q, k} \leq P_{\max }, \forall(q, k)\right\}$ stands for the feasible set of transmit power vectors under the specific power requirements. We extend a technique presented in [12] to narrow down the possible solutions at the non-convex problem of (12). Given a factor $\varepsilon>1$ and power allocation profile $\mathbf{p}$ :

$$
\begin{array}{r}
R_{q}(\varepsilon \mathbf{p}) \triangleq R_{q}\left(\varepsilon P_{1,1}, \ldots, \varepsilon P_{Q, K}\right)= \\
\log \left(1+\frac{\sum_{\dot{q}=1}^{Q} \sum_{k=1}^{K} P_{\dot{q}, k}\left(\varsigma_{m, \dot{q}, k}^{m, q}\right)^{2}}{\frac{\sigma_{0}^{2}}{\varepsilon}+\sum_{\dot{m}} \sum_{\dot{q}=1}^{Q} \sum_{k=1}^{K} P_{\dot{q}, k}\left(\varsigma_{\dot{m}, \dot{q}, k}^{m, q}\right)^{2}}\right) \\
>R_{q}(\mathbf{p})
\end{array}
$$

for all BSs $q$ and any cluster size $Q$. Since the total cluster sum rate is $R(\varepsilon \mathbf{p})=\sum_{q=1}^{Q} R_{q}(\varepsilon \mathbf{p})$, we have also that $R(\varepsilon \mathbf{p})>$ $R(\mathbf{p})$. According to the above we may construct the following.

Lemma 1: If a UT power constraint exists, at least one element of vector $\mathbf{p}$ must be equal to that constraint to maximize cluster sum rate $R$.

The maximization problem of the cluster sum rate can be split into two subproblems: Maximise cluster sum rate by providing more power to UTs that are: 1) closer to their respective BS (defined as UT-w.r.t.-cell location dependance) and, 2) closer to the center of their respective cluster (defined as UT-w.r.t.-cluster location dependance). Considering (10), the achievable sum rate is increased when the desired received power in the cluster is maximized while the undesired received power is minimised at the same time. In addition to that, the sum of all BS contributing rates in the cluster $\left(\sum_{q=1}^{Q} R_{q}=R\right)$ should also be maximized. From (9) we observe that the cluster sum rate depends on the product of the squared path loss coefficients associated to the UT-BS paths with the respective UT transmitting powers. Moreover: 1) the squared path loss coefficients at the numerator are a function of the UTs' distance from their same cell BS while, 2) the squared path loss coefficients at the denominator depend on the distance of the UTs' same cell BS to the BSs of the other clusters. According to that, the UTs close to their respective BSs and close to the center of their respective cluster, contribute more on the desired power and cause less interference to the neighbouring clusters . Hence, if an optimal power vector $\mathbf{p}^{\star}$ exists, the power element(s) that is(are) equal to the power constraint, so at to maximize sum rate according to Lemma 1, should refer to the UT(s) located closer both to its(their) respective BS and to the centre of its(their) respective cluster.

\section{A. Power Allocation Profiles}

Two general power allocation profile vectors are introduced ( $\mathbf{p}_{\text {cell }}$ for the UT-w.r.t-cell and $\mathbf{p}_{\text {cluster }}$ for the UT-w.r.t.-cluster location dependance) which can be combined to provide the set of the feasible optimal UT power allocation profiles. The united power allocation profile vector $\hat{\mathbf{p}}$ is a weighted combination of profiles $\mathbf{p}_{\text {cluster }}$ and $\mathbf{p}_{\text {cell }}$ controlled by parameter $\nu$ (with $0 \leq \nu \leq 1$ ). Higher values of $\nu$ refer to a power allocation more favoured by the UT-w.r.t.-cluster location dependance power profile and vice-versa:

$$
\hat{\mathbf{p}} \triangleq \nu \mathbf{p}_{\text {cluster }}+(1-\nu) \mathbf{p}_{\text {cell }}
$$

Four parameters $\left(\alpha_{1,2,3,4}\right.$ for $\mathbf{p}_{\text {cluster }}$ and $\beta_{1,2,3,4}$ for $\left.\mathbf{p}_{\text {cell }}\right)$ are defined to control the curve of each general power profile with:

$$
\begin{aligned}
& 0 \leq \alpha_{1}, \beta_{1} \leq 0.5 \text { - edge-UTs allocated with } P_{\min } \\
& 0 \leq \alpha_{2}, \beta_{2} \leq 0.5 \text { - centre-UTs allocated with } P_{\max } \\
& 0 \leq \alpha_{3}, \beta_{3} \leq 1-P_{\min }=\alpha_{3} P_{\max } \text { or } \beta_{3} P_{\max } \\
& \alpha_{4}, \beta_{4}=\{-1,0,+1\} \text { - define the power allocation curve }
\end{aligned}
$$

with $\left(\alpha_{1}+\alpha_{2}\right),\left(\beta_{1}+\beta_{2}\right) \leq 0.5$. Terms edge-,centre- refer to the edge or the center of either the cell or cluster respectively depending on the general power profile we refer to. Moreover, $\alpha_{4}, \beta_{4}=0$ refers to a linear power allocation curve while $\alpha_{4}, \beta_{4}= \pm 1$ to sinusoidal curves (Figure 2).

The power of a UT will be a function of distance $s$ from its respective BS and hence the general power allocation profile vector $\mathbf{p}_{\text {cluster }}$ will have elements:

$$
\left\{\begin{array}{cc}
P_{\min } & Q \operatorname{ISD}\left(1 / 2-\alpha_{1}\right) \leq s \leq \frac{Q \mathrm{ISD}}{2} \\
P_{0}(s) & a_{4}=0, \alpha_{2} Q \mathrm{ISD} \leq s \leq Q \mathrm{ISD}\left(1 / 2-\alpha_{1}\right) \\
P_{+1}(s) & a_{4}=+1, \alpha_{2} Q \mathrm{ISD} \leq s \leq Q \operatorname{ISD}\left(1 / 2-\alpha_{1}\right) \\
P_{-1}(s) & a_{4}=-1, \alpha_{2} Q \mathrm{ISD} \leq s \leq Q \mathrm{ISD}\left(1 / 2-\alpha_{1}\right) \\
P_{\max } & 0 \leq s \leq \alpha_{2} Q \operatorname{ISD}
\end{array}\right\}
$$

where the various power functions are defined as $P_{0}(s) \triangleq P_{\text {min }}+P_{\text {diff }} \frac{\left|s-Q \operatorname{ISD}\left(\frac{1}{2}-\alpha_{1}\right)\right|}{\left.Q \operatorname{ISD}-\alpha_{1}-\alpha_{2}\right)}, \quad P_{+1}(s) \triangleq$ $P_{\text {min }}+P_{\text {diff }} \sin \left(\frac{\frac{\pi}{2}\left|s-Q \operatorname{ISD}\left(\frac{1}{2}-\alpha_{1}\right)\right|}{Q \operatorname{ISD}\left(\frac{1}{2}-\alpha_{1}-\alpha_{2}\right)}\right), P_{-1}(s) \triangleq 2 P_{0}-P_{+1}$ with $P_{\text {diff }}$ denoting the difference $P_{\max }-P_{\min }$. An example of the $\mathbf{p}_{\text {cluster }}$ profile is illustrated in Figure 2. Similar expression and representation exists for the $\mathbf{p}_{\text {cell }}$ profile where QISD and $\alpha$ parameters in (16) will be replaced by ISD and $\beta$ 


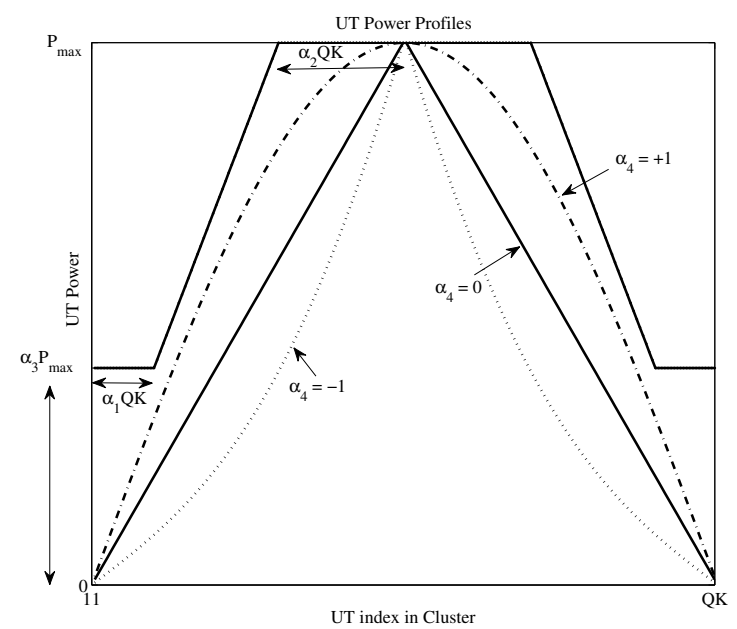

Fig. 2. $\quad \mathbf{p}_{\text {cluster }}$ power allocation profile and parameters $\alpha_{1,2,3,4}$.

respectively in that case. The combination of the two general power allocations by $\nu$ and parameters $(\alpha, \beta)_{1,2,3,4}$ provide a mathematically tractable set of all the feasible power profiles that can maximize the cluster sum rate.

\section{NUMERICAL RESUlts AND OBSERVATIONS}

For interpreting the information theoretic results into realworld systems we assume the practical scenario described in [7]. The results for the cluster sum rate have been produced by generating the corresponding system and applying (9) and they have been verified by running Monte Carlo simulations to generate random fading coefficients for various system snapshots. The per cluster sum rates for the verification are obtained by finding the average over a large number $J$ of system snapshots using $R_{\text {sim }}=\frac{1}{J} \sum_{1}^{J} \log \left(\frac{\operatorname{det}\left(\mathbf{H} \boldsymbol{\Lambda}_{\mathbf{x}} \mathbf{H}^{\dagger}+\boldsymbol{\Lambda}_{\mathbf{z}}\right)}{\operatorname{det}\left(\boldsymbol{\Lambda}_{\mathbf{z}}\right)}\right)$. The simulated linear cellular system contains clusters of cells with 1 to 20 cells each. 100 UTs are uniformly distributed across each cell. Results of the normalised cluster sum rate (averaged cluster sum rate over the cluster size) and of the BS contribution rate distribution (when $Q=20$ ) for three different density systems are provided. Various UT power profiles were applied and their effect on the sum rate was investigated.

The following general observations were made. The optimal UT power allocation strategy in any studied case, so as to maximise the cluster sum rate, is to give maximum priority to the UT-w.r.t-cell (instead of UT-w.r.t-cluster) location dependence (e.g. $\nu=0$ ). In addition, it is preferable to allow only the UTs that are at any time very close to their BS to transmit at their maximum allowed power while limiting all the other UT powers to $P_{\min }$ (ideally $P_{\min }=0$ ). On the following although we provide the maximum achievable sum rate (for $\left.\nu=0, \beta_{1} \rightarrow 0.5\right)$ we also examine the behaviour of the achievable sum rate when UT-w.r.t-cluster location dependence is considered in which case the rate will be allocated more fairly among the UTs. When $\nu \neq 0$, it is better to restrain the cluster edge UTs to low power while allowing maximum
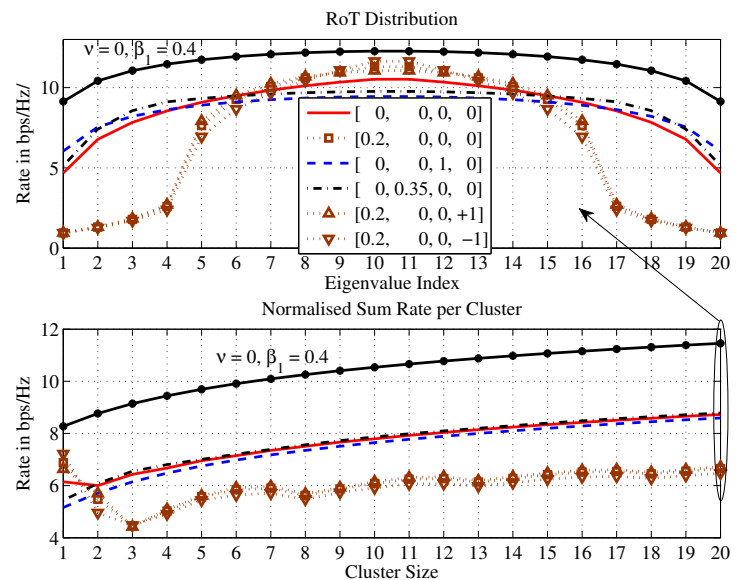

Fig. 3. Normalised cluster sum rate and BS contribution rate distribution of a dense system for various UT power profiles. Legend shows the various $\left[\alpha_{1}, \alpha_{2}, \alpha_{3}, \alpha_{4}\right]$ combinations.

power to cluster centre UTs. It is not optimal though to restrain many cluster edge UTs to the minimum power as the positive effect of reducing their interference to neighbouring clusters is in most cases overlapped by the negative effect of less desired power in the cluster (keep $\alpha_{1} \simeq 0$ in any case).

We first consider a dense system with ISD $=100 \mathrm{~m}$ and $\eta=2$. In that case the thermal noise variance becomes a minor parameter when compared to the other powers and can be neglected. Moreover, when constant power profiles are applied (every UT is allocated the same power) from (9) it is occurs that sum rate is totally independent from the UT powers. Figure 3 illustrates results for various UT power allocation profiles. When $\nu \neq 0$, it can be observed that allocating $P_{\max }$ to high percentage of UTs is preferred for maximising sum rate (i.e. $\alpha_{2}=0.35$ instead of 0.5 , although the latter provides fairer cell RoT distribution). Moreover, it is better to keep the value of $P_{\min }$ as low as possible $\left(\alpha_{3} \simeq 0\right)$ which effectively means to give low power to the edge cluster UTs. It is noted that for $\nu=0$ a significant improvement on the cluster sum rate is observed. In Figure 4, a sparse system with ISD $=6 \mathrm{Km}$ and $\eta=3.5$ is considered. In this scenario, we should allocate as much power as possible to the UTs $\left(\alpha_{2} \simeq 0.5\right.$ or $\alpha_{3} \simeq 1$ when $\left.\nu=1\right)$. The above means that in a sparse environment the cluster edge effects become negligible and thus, there is no need for power control, i.e. letting UTs to transmit at their maximum power is the best choice. Furthermore, it is observed that constant UT power allocation profiles provide fairer solutions for cell RoT distribution and in these cases it is valid to state that the normalised cluster sum rate is equivalent to the per-cell sum rate. Figure 5 depicts results for a medium density system with ISD $=2 \mathrm{Km}$ and $\eta=3$. Here, for $\nu \neq 0$, allocating maximum available power is again optimal when the cluster size is large enough. On the other side, for relatively small cluster size, the optimal power allocation set parameters of the dense systems are also preferable here. We can observe 

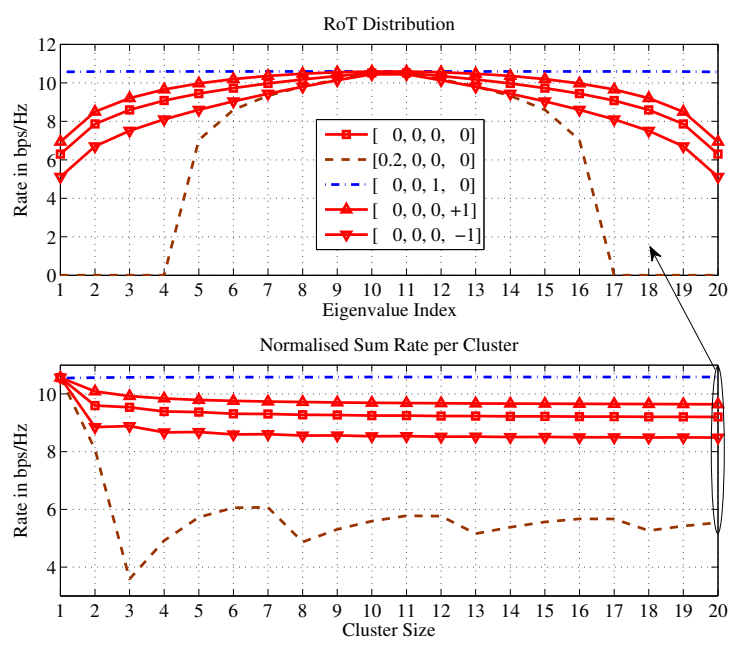

Fig. 4. Normalised cluster sum rate and BS contribution rate distribution of a sparse system for various UT power profiles.
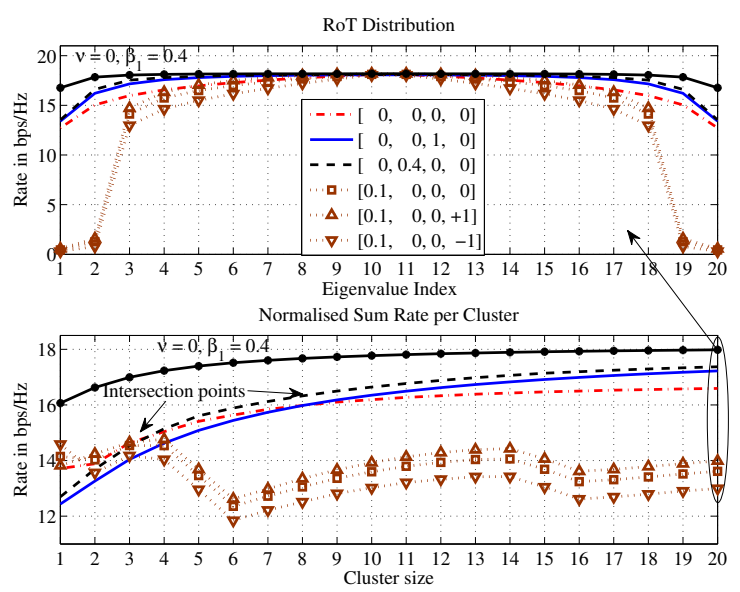

Fig. 5. Normalised cluster sum rate and RoT distribution of a medium density system for various UT power profiles.

various intersection points which define for which cluster sizes each profile becomes preferable than the other. It is noted that the position of these intersection points on the $\mathrm{x}$-axis (cluster size) depends always on the combination of parameters $\alpha_{2,3,4}$. For $\nu=0$, achievable cluster sum rate is increased and at the same time the cell RoT distribution becomes fairer.

Based on the work reported in [8], we provide the maximum achievable per-cell sum rate for each of the three density systems presented above when using a HR. When allocating $P_{\max }$ (here $P_{\max }=200 \mathrm{~mW}$ ) to each UT the rate of the 1) dense system reaches approximately to $30.5 \mathrm{bps} / \mathrm{Hz} / \mathrm{Cell}$, 2) medium density system to $18.3 \mathrm{bps} / \mathrm{Hz} / \mathrm{Cell}$ and 3) sparse system to $10.6 \mathrm{bps} / \mathrm{Hz} / \mathrm{Cell}$. These results in comparison with the ones illustrated in Figures 3-5 indicate the rate differences between the global and the clustered BS cooperation case. It is obvious that the dense systems suffer severely from the inter-cluster interference (achieving $<40 \%$ of the maximum capacity even for $Q=20$ cells per cluster) while medium density systems are proved to be the most viable case for a clustered system design (can achieve $>80 \%$ of the maximum capacity even with less than 5 cells per cluster). We should note as well that cooperation between BSs, in general, does not increase the achievable sum rate of very sparse systems.

\section{CONCLUSION}

In this paper the sum rate for the uplink of a cluster cellular system is investigated. Considering a linear cellular model we formulate the problem by capturing the inter-cluster interference into an information theoretic sum rate analysis. For an interference allowance scheme the optimization of the cluster sum rate problem under UT power constraints is analysed. After providing a generic UT power allocation profile we investigate the effect of power control on the no isolation clustered system uplink and compare the results to these of the BS global cooperation case. It is observed that cell-based is preferable to the cluster-based UT power control and that medium density systems significantly benefit from the clustered BS joint-processing and reach closer to the capacity of the respective HR systems. Finally, the distribution of the BS contribution rates is introduced as a metric that can be proved useful for the positioning of the BSs in a scenario where the BSs could, by request, be distributed non-uniformly over the clusters and it could be the case for a future work.

\section{REFERENCES}

[1] A. Wyner, "Shannon-theoretic approach to a Gaussian cellular multipleaccess channel," Information Theory, IEEE Transactions on, vol. 40, no. 6, pp. 1713-1727, Nov 1994.

[2] O. Somekh and S. Shamai, "Shannon-theoretic approach to a Gaussian cellular multiple-access channel with fading," Information Theory, IEEE Transactions on, vol. 46, no. 4, pp. 1401-1425, Jul 2000.

[3] N. Letzepis, "Gaussian cellular muptiple access channels," Ph.D. dissertation, Institute for Telecommunications Research, University of South Australia, Dec 2005.

[4] M. Bacha, J. Evans, and S. Hanly, "On the capacity of mimo cellular networks with macrodiversity," in Communications Theory Workshop, 2006. Proceedings. 7th Australian, 1-3 Feb. 2006, pp. 105-109.

[5] O. Somekh, B. M. Zaidel, and S. Shamai, "Sum rate characterization of joint multiple cell site processing," Information Theory, IEEE Transactions on, vol. 53, no. 12, pp. 4473-4497, Dec 2007.

[6] M. I. E. Katranaras and R. Hoshyar, "Sum rate of linear cellular systems with clustered joint processing," IEEE 69th Vehicular Technology Conference, (VTC'09), pp. 1-5, 2009.

[7] M. I. E. Katranaras and C. Tzaras, "framework to compare the uplink capacity of the cellular systems with variable inter site distance," IEEE 19th International Symposium on Personal, Indoor and Mobile Radio Communications (PIMRC'08),, pp. 1-5, 2008.

[8] E. Katranaras, M. Imran, and C. Tzaras, "uplink capacity of a variable density cellular system with multicell processing," IEEE Transactions on Communications, vol. 57, no. 7, pp. 2098-2108, July 2009.

[9] D. Tse and P. Viswanath, Fundamentals of Wireless Communications. University Press, Cambridge, 2005.

[10] S. Jayaweera and H. Poor, "Mimo capacity results for rician fading channels," in IEEE Global Telecommunications Conference, 2003. GLOBECOM '03, vol. 4, 1-5 Dec. 2003, pp. 1806-1810.

[11] A. Goldsmith, Wireless Communications. Cambridge University Press, 2005.

[12] C. S. Chen and G. E. Oien, "Optimal power allocation for twocell sum rate maximization under minimum rate constraints," Wireless Communication Systems, ISWCS'08, IEEE International Symposium on. 